\title{
How Could This Happen?
}

\section{Narrowing Down the Contagion of COVID-19 and Preventing Acute Respiratory Distress Syndrome (ARDS)}

\section{Wilfried Allaerts ${ }^{1}$}

Received: 26 March 2020 / Accepted: 10 April 2020 / Published online: 25 April 2020

(c) Springer Nature B.V. 2020

\begin{abstract}
In this rapid commentary, a mini-review is given of the present state-of-knowledge regarding the etiology and epidemiology of the new coronavirus 2019-nCoV and the risks for developing Acute respiratory distress syndrome (ARDS). The available knowledge on the viral genomics, molecular biology and pathogenicity of viruses of the Coronaviridae family and other Nidovirales, forms a helpful template for understanding the present pandemic outbreak. However, important questions remain unanswered about the underlying mechanism causing the very high case fatality ratios (CFR) and mechanisms regarding severe reactions like ARDS, fatal cardiac and renal failures, associated with a number of important comorbidity factors. Immunological reactions to lung alveoles in particular (involving lung macrophages and alveolar epithelial cell damage) in late phase ARDS in SARS-like CoV diseases, so far may not have received enough attention. Finally a shortlist of questions for high priority further research is suggested.
\end{abstract}

Keywords COVID-19 (2019-nCoV) - SARS · MERS · Acute respiratory distress syndrome $\cdot$ Angiotensin converting enzyme $2 \cdot$ Lung immunopathology

\section{Introduction}

Since the outbreak of the 2019 novel coronavirus (named 2019-nCoV by the World Health Organization) (Wu et al. 2020), also named coronavirus disease 2019 (Covid19) or SARS-CoV-2, the disease has spread to a global pandemic. The effects of this pandemic not only on public health and health care institutions, but also on nearly every aspect of social life and worldwide economy is unprecedented in postmodern history. The speed of its distribution, since the discovery on 30 December 2019

Wilfried Allaerts

1 Biological Publishing A\&O and Immunology Department, Erasmus MC, Rotterdam,

The Netherlands 
of a new virus in the bronchioalveolar lavage (BAL) (samples taken from the lung content) of a patient at Wuhan Jinyingtan Hospital (Hubei province, China) (WHO 2020) until today - which is less than 3 months - has caused a shockwave in a number of severely struck countries. Unfortunately, it may have caused a lot of confusion in the public media too. Especially the high rate of fatalities, following the incidence of acute respiratory distress syndrome (ARDS) has raised the stakes of national health authorities up to the highest levels of vigilance and promulgation of the most extreme measures.

This paper aims at summarizing the information already available concerning the etiology, genomic background and epidemiology of COVID-19. In particular, similarities and differences observed in related species of the Coronaviridae (order Nidovirales), especially of the genus Betacoronavirus, and most predominantly the SARS-related Rhinolophus bat coronavirus (SARSr BaCoV HKU3) and SARSrelated human coronavirus (SARS CoV Urbani) (International Committee on Taxonomy of Viruses 2012) are helpful to understand the pathogenicity of this new coronavirus. The COVID-19 pandemic strongly recalls characteristics of the severe acute respiratory syndrome (SARS) pandemic of 2002-2003, and therefore has been called SARS-CoV-2 in many studies too. However, especially the differences between SARS, Influenza and COVID-19 are crucial to comprehend the divergent epidemiology and pathogenicity of these diseases (Wu et al. 2020; Weiss and Leibowitz 2011). Moreover, the etiology of pneumonia in these diseases and important correlations between SARS-related and COVID-19-related ARDS are summarized in Sect. 3.

From the early phases of the outbreak of COVID-19 it has been noted that people above the age of 60 years belong to a high risk group for developing fatal organ failure, especially due to lung damage. In some countries, the age level for high risk patients was set at 70 (e.g. in the United Kingdom). Whereas the overall Case Fatality Ratio (CFR) from a large Chinese study group was determined as $2.3 \%$ (The Novel Pneumonia Emergency Response Epidemiology Team 2020), it was clear that higher CFR's were found in elder people. An alternative to expressing the fatality of the coronavirus diseases in terms of CFR is to calculate the standard expected years of life lost (SEYLL index) (Salamatbakhsh et al. 2019). Important co-morbidities were ascribed to hypertension (CFR 6.0\%), diabetes (CFR 7.3\%), cardiovascular disease (CFR 10.5\%) and age above 70 (CFR 10.2\%) (Novel Pneumonia Emergency Response Epidemiology Team 2020). Due to the association of at least two of these co-morbidities with the widely prescribed use of ACE-inhibitors-for instance used for blocking the angiotensin-receptor (ATR) in order to lower blood pressure (for hypertensive and cardiac patients altogether)-, the risks of developing ARDS in these patient groups should be considered with highest priority. So far, only animal experiments have documented that ACE-inhibitors can significantly increase mRNA expression of cardiac ACE2 (Ferrario et al. 2005), but some already caution for using ACE inhibitors as potential risk factor (Sommerstein 2020). It is concluded that all possible public health protection measures for these category of patients, in addition to the infectivity measures against spreading the COVID-19 virus among all age groups, need our highest priority attention. 


\section{Etiology and Genomic Background of COVID-19, SARS and MERS}

Initial genomic analysis has shown a close evolutionary association with the SARSlike bat coronaviruses ( $\mathrm{Wu}$ et al. 2020). This relationship is confirmed by $96 \%$ genomic similarity of the full-length genome sequence of the COVID-19 virus with a SARS-like coronavirus strain derived from Rhinolophus bat (BatCoV RaTG13) (WHO 2020). Only recently it was shown that genomic recombination from the Chinese Greater Horseshoe bat (R. sinicus) was at the onset of the SARS outbreak (Lau et al. 2015). Similar to the SARS outbreak of 2002-2003, the viral tropism towards the respiratory system is striking, but the timing of the highly infective phases of the disease are also very different from SARS. The very high human-to-human infectivity of COVID-19 and resulting pneumonia, has been documented following close surveillance of pneumonia in a family cluster, early during the Wuhan epidemic (Chan et al. 2020).

In the etiology of late phase severe lung damage, an important role is played by the angiotensin converting enzyme 2 (ACE2) as well as by lymphocytic inflammatory responses (Weiss and Leibowitz 2011). It has been known for a while that ACE2 is involved in acute lung injury in mice (Imai et al. 2008) and that ACE2 is a receptor of the spike (S) glycoprotein of both Mouse Hepatitis Virus (MHV2) - also a Betacoronavirus causing virulent pneumonia-and SARS-related CoV (Weiss and Leibowitz 2011). The importance of this ACE2 receptor for cellular infectivity of the virus is strengthened by the finding that no amino acid substitutions have been found in the corresponding genome region of 2019-nCoV, compared with the receptor-binding motifs that directly interact with human ACE2 as recognized in the SARS-CoV genome (Wu et al. 2020). Meanwhile, it has been demonstrated that 2019-nCoV uses the same ACE2 receptor-similar to the SARS-coronavirus receptor ACE2 - and the cellular protease TMPRSS2 for entry into target cells (Hoffmann et al. 2020). Moreover, ACE2 expression is very predominant in both Type 1 and Type 2 alveolar pneumocytes of the human lung, as well as in the intestine and kidney (Turner 2015).

In contrast to the former examples of Betacoronaviruses (MHV-2, SARSrCoV and $2019 \mathrm{nCoV}$ ), in the middle east respiratory syndrome (MERS) (Toosy and O'Sullivan 2017) a different cell entry of the MERS-related coronavirus (MERS$\mathrm{CoV}$ ) was discovered: namely the cellular receptor dipeptidyl peptidase 4 (DPP4, also known as CD26) (Raj et al. 2013). The epidemiology of MERS is quite different from that seen in SARS (Toosy and O'Sullivan 2017) and also from COVID19. The receptor for viral entry, DPP4 (CD26) is for instance expressed in cells of the monocyte-macrophage lineage. Moreover, DPP4 is expressed on epithelial and endothelial cells of most human organ tissues (in ex vivo studies using human culture lines), suggesting this may account for the multisystem clinical spectrum of MERS-CoV infection (Turner 2015). Although also MERS has been associated with a coronavirus lineage present in Vesper bats (in particular Pipistrellus hesperidus, Vespertilionidae) in Uganda, there was a high divergence of the $\mathrm{S}$ protein nucleotide sequence compared with a MERS-CoV S protein sequence (Anthony et al. 2017). This suggests that the viruses are different and-although bats are the plausible 
evolutionary source of MERS CoV-another intermediate mammalian reservoir species is probably responsible for transmission of MERS to humans (Turner 2015). This appears to be the wild population of Dromedary camels in the Arabian Peninsula, as well as in North and East Africa. It is suggested that the limited geographic distribution of MERS-CoV is related to the distribution of these dromedary camels (DCs). When DCs are infected, MERS-CoV causes only a mild, transient upper respiratory tract disease (Turner 2015).

Genomic analysis of the 2019-nCoV and closely related coronaviruses already has revealed a number of important differences (Wu et al. 2020). In order to understand the impact of these differences on pathogenicity and epidemiology, it is important to have access to the genomic structure of the virus RNA (See Fig. 1a) (Wu et al. 2020; International Committee on Taxonomy of Viruses 2012). First a distinction is made in genes coding for structural (glyco)proteins (Fig. 2) forming a common backbone in many groups of the Nidovirales: these include the spike glycoprotein $(\mathrm{S})$, the membrane glycoprotein $(\mathrm{M})$, the envelope protein $(\mathrm{E})$, the nucleocapsid protein $(\mathrm{N})$ and the hemagglutinin-esterase glycoprotein (HE). However, the latter HE is absent from both SARSr human CoV, and from SARSr bat CoV as well as from 2019-nCoV (Wu et al. 2020) (Fig. 1b). In other members of the Betacoronavirus genus, like in Human coronavirus HKU1 (International Committee on Taxonomy of Viruses 2012), HE is important for reversible attachment of virions to O-acetylated sialic acids, by acting both as a lectin and as a sialate- $O$-acetylesterase. This however is not the case in neither SARS nor 2019-nCoV. Also for the relatively innocuous Human coronavirus OC43 (causing only a mild cold), it is suggested that 9 - $O$-acetylated sialic acid is the primary attachment factor, and not the ACE2 receptor (International Committee on Taxonomy of Viruses 2012). Sialic acid plays an important role in cellular recognition and ganglioside metabolism, and is found as a common modification at the outside of glycoproteins. For instance, the $9-O$-acetylation of these sialoglycans has been shown to play a role in host-pathogen interactions with functional implications in the immune system (like impaired binding of influenza A and B, but at the same time creating a receptor for influenza C) (Baumann et al. 2015).

Beside the structural (glyco)proteins, an important number of non-structural proteins are generated from the virus genome, with a variety of functions like degradation of host mRNAs, polyprotein processing and association with replicase/transcriptase complexes (International Committee on Taxonomy of Viruses 2012). In the so-called open reading frames (ORF $1 \mathrm{ab}$ and ORF 1a), both situated at the 5'-terminus of the genome of 2019-nCoV—as well as in most Betacoronavirus genomes-, a number of non-structural proteins (nsps) are encoded (International Committee on Taxonomy of Viruses 2012) (Fig. 1a). In 2019-nCoV the first ORF represents $67 \%$ of the entire genome, being transcribed into so-called polyproteins that are further processed into $16 \mathrm{nsps}$ (Wu et al. 2020). Beside a number of differences in the nsps encoding ORF, also in the eight so-called accessory proteins that are encoded in ORF's near the 3 ' end of the genome, important differences have been noted between 2019-nCoV and SARS-like coronaviruses (Wu et al. 2020). However, a lot of research and time will be needed to understand how these differences may 

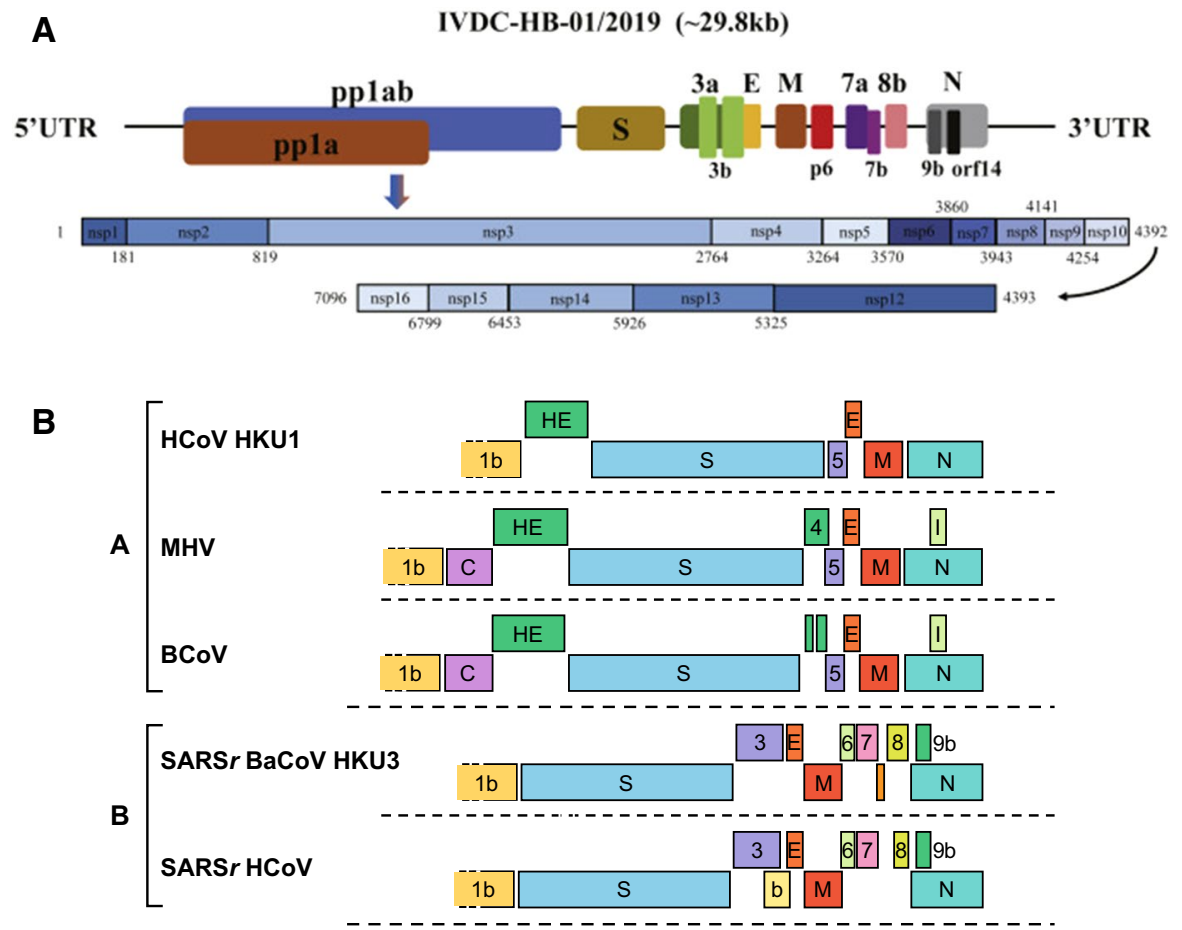

Fig. 1 a Schematic diagram of genome organization and the encoded proteins for 2019-nCoV (based on genome of Wuhan/IVDC-HB-01/2019 virus) (@ 2020 Elsevier Inc.) (adapted from Wu et al. 2020). ppla, pplab polyprotein 1a, resp $1 \mathrm{ab}, n s p$ non-structural protein. See main text for clarification of used symbols. b Schematic genome organization in the A- and B-group, of genus Betacoronavirus (@ 2012 International Committee on Taxonomy of Viruses; publ. Elsevier Inc.) The figure shows the genomes of a number of representative species (only the 3 '-terminal genomic regions downstream of ORF1b are shown. Mark the absence of the HE region (coding for hemagglutinin esterase enzyme) in SARS related bat and human CoV (see Fig. 2 for enzyme codes and also main text for abbreviations of virus names)

affect the pathogenicity and epidemiology of 2019-nCoV: this precious time, unfortunately, might not be available for a large proportion of our population.

\section{Risk Factors for Acute Respiratory Distress Syndrome (ARDS)}

Because the 2019-nCoV also uses the ACE2 receptor for entry in the host-like the SARS-related $\mathrm{CoV}$ - and because the very dangerous reactions this interaction may cause in the human lung, a lot of information is already present in medical publications.

The pharmacology of the angiotensin converting enzyme 2 (ACE2), its physiological role and relationship with acute respiratory distress syndrome (ARDS), already have been studied extensively (Imai et al. 2008). ARDS is known as a very severe, life-threatening form of acute lung injury (ALI). Many pathogenic conditions have been found to trigger ARDS, not only SARS and COVID-19, but also 


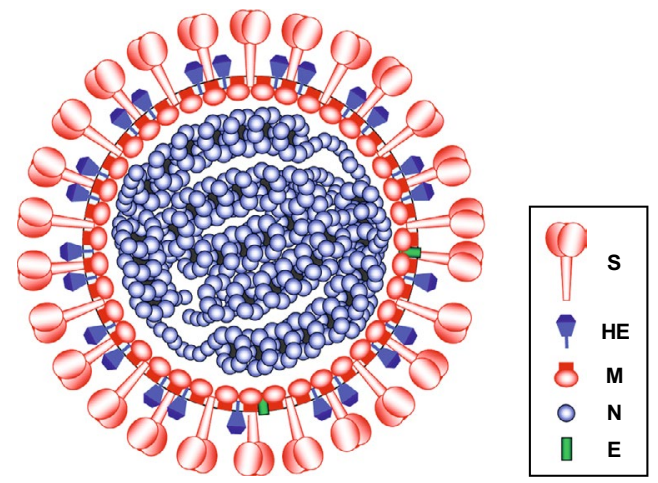

Fig. 2 Schematic representation of a lineage A betacoronavirus virion (C) 2012 International Committee on Taxonomy of Viruses; publ. Elsevier Inc.) S: the spike (glyco) protein (responsible for binding to the host cell receptor ACE2 in SARS CoV and 2019-nCoV); HE: Hemagglutinin Esterase (not expressed in B lineage, see also Fig. 1b). $M$ membrane glycoprotein, $N$ nucleocapsid protein, $E$ envelope protein. Both $\mathrm{S}$ and HE proteins are heavily glycosylated, $\mathrm{M}$ contains a small number of glycans, concerning the species. Moreover, the $\mathrm{S}$ and $\mathrm{E}$ proteins are palmitoylated, providing them their hydrophobic features

pathogens like Anthrax, Spanish flu virus and H5N1 avian influenza virus (Rubenfeld et al. 2005). However, the association of binding of the coronavirus spike glycoprotein (S) with ACE2, and subsequent catalytic cleavage in the endosome (at low $\mathrm{pH}$, involving cathepsin $\mathrm{L}$ and a second leu-peptin-sensitive-like cleavage in the case of SARS-CoV) (Weiss and Leibowitz 2011), so far is characteristic for only a few members of the Betacoronavirus genus (see also Sect. 2).

The pathogenic effects of coronavirus entry using the ACE2 receptor in for instance lung epithelial cells appear to follow two paths: on the one hand, spikereceptor interaction causes downregulation and abolishment of the protective function of ACE2, resulting in local enhancement of angiotensin II production and unrestricted stimulation of the angiotensin receptor $\left(\mathrm{AT}_{1} \mathrm{R}\right)$ (Imai et al. 2008) (Fig. 3). On the other hand, the binding of ACE2 to SARS-CoV spike glycoprotein induces so-called syncytia-formation and transformation into large multinucleated cells, as observed in clinical SARS infections (Imai et al. 2008) and also in post mortem samples taken from a 50-year old male patient from Wuhan (WHO 2020). It was already suggested that severe lung damage, especially in the late phase of SARS, was largely 'immunopathological in nature' (Weiss and Leibowitz 2011). In early phase fatal lung disease, histological examination showed hyaline membrane formation (indicative of ARDS) (World Health Organization 2020) and loss of lung epithelial cells (or pneumocytes), resulting alveolar damage and mixed cellular infiltrates of lymphocytes (Weiss and Leibowitz 2011). Often also multinucleated giant cells, carrying markers for both macrophages and pneumocytes were present (Weiss and Leibowitz 2011), indicating the involvement of macrophage scavenging. Also in 2019-nCoV infections, these histological features were observed in post mortem specimen from lung (apart from the demonstration of lineage markers) (World Health Organization 2020). In the late phase of SARS (phase III, 10-15 days after the onset of illness), also type II pneumocyte hyperplasia, squamous metaplasia and 


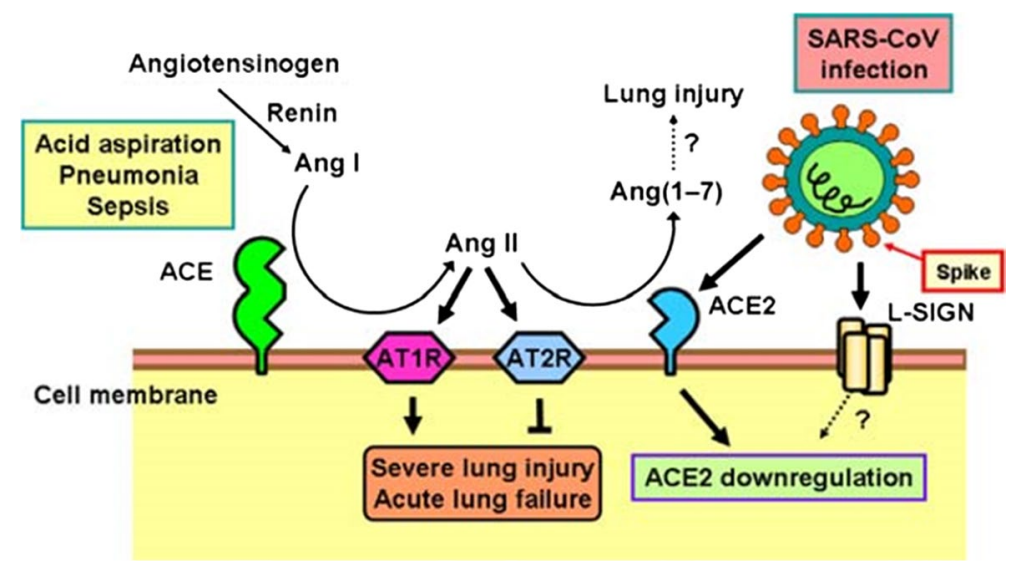

Fig. 3 Schematic diagram representing the role of the renin-angiotensin system in acute lung failure and proposed SARS-CoV action during infection (ㄷ 2008 The Physiological Society) (adapted from Imai et al. 2008)."In acute lung injury, such as acid aspiration, pneumonia or scepsis, the generation of Ang II from Ang I is enhanced by ACE, and Ang II induces acute lung failure through stimulation of $\mathrm{AT}_{1} \mathrm{R}$ while ACE2 and AT2R negatively regulate this pathway and protect from acute lung failure. In contrast, SARS-CoV infection is mediated through binding of the SARS Spike Protein to ACE2 (...) and downregulates the protective molecule ACE2, thus leading to severe lung injury and acute lung failure" (cited from Imai et al. 2008) (A possible, alternative route via L-SIGN, i.e. the liver/lymph node-specific intercellular adhesion molecule-3-grabbing nonintegrin, may result in a similar clinical outcome)

bronchiolitis obliterans were found in histological examination. These features are consistent with the late, irreversible obstruction of the bronchioles (Weiss and Leibowitz 2011). The worsening of clinical progression, simultaneously with declining viral loads and markedly elevated cytokine levels, the so-called cytokine-storm (during SARS phase III, but also seen in COVID-19), corroborate the conclusion that these late pathological effects are the result of a fatal overreaction of the immune system (Weiss and Leibowitz 2011) (see below).

Similarly, in a large Chinese study, also other groups with impaired lung functioning, like patients with chronic obstructive pulmonary disease (COPD), have shown to constitute an important risk with high co-morbidity rate when infected with this new coronavirus (Guan et al. 2020). To these potential risk groups, also patients with fibrotic lung developments following several (auto) immune disorders (like rheumatoid arthritis, systemic lupus erythematosus [SLE], scleroderma, etcetera) and other etiologies causing impaired lung functioning, may be added. The importance of resident lung macrophages in keeping a tolerant immune state of the lungs, in order to strongly inhibit local $\mathrm{T}$ cell responses, has been noted for a considerable time (Holt 1994). During inflammation, a 'protective' immune reaction is raised, involving $\mathrm{T}$ cell reactions directed to the lungs (Holt 1994). However, obviously, insufficient knowledge is available enabling us to link the activated characteristics of a patient's immune system and the risk of developing ARDS following infection with $2019 \mathrm{nCoV}$. Similar to the risk of developing allergic disorders, too little information is yet applicable on the importance of a T-helper-2-type responsiveness 
(tolerogenic) versus a T-helper-1-type responsiveness (inflammatory reaction) in the immune reactions to these viral infections (Allaerts and Chang 2017).

After some initial optimism, suggesting the potential usefulness of ACE-inhibitors and/or $\mathrm{AT}_{1} \mathrm{R}$ inhibitors to build protection against severe lung damage (Weiss and Leibowitz 2011), recent experimental findings have raised some doubts (Ferrario et al. 2005) or cautioned against its use as prophylactic medication (Sommerstein 2020). Given the increased vulnerability of patients with age and the widespread use of ACE-inhibitors, more research into the epidemiological relation between the use of ACE-inhibitors and 2019-nCoV-related ARDS is needed. To our opinion, this should become prioritized in global public health research related to COVID-19.

During the SARS pandemic, it appeared that the SARS-CoV spike protein (S) was a primary factor in determining the virulence of the attack, "beyond its role in viral attachment and cell entry"(Weiss and Leibowitz 2011). Experiments in vitro using mouse heart cells, moreover, have shown the downregulation of ACE2, following viral inflammation and viral replication in the myocardium cells (Weiss and Leibowitz 2011). This suggests that also cardiac infection could contribute to SARS mortality (Oudit et al. 2009). Similarly, ACE-inhibition may influence renal function and interstitial renal damage following SARS infection (Hamming 2008). Also during the COVID-19 epidemic in China, chronic kidney diseases also have constituted a high co-morbidity factor comparable to the comorbidity associated with diabetes, following the highest comorbidity associated with hypertension (Guan et al. 2020). The combination of lung, cardiac and/or renal failure creates a fatal mixture at intensive care hospitals, when SARS patients often were already in their third week of illness and symptoms of viral infection were declining (Weiss and Leibowitz 2011).

\section{Epidemiological Lessons from Other Coronaviridae and New Viral Diseases}

One of the cardinal questions related to this new burden of coronavirus-related diseases is how humanity will deal with it, apart from the questions of how recurrent pandemics would influence the world economy, the demography and migration patterns and finally also the world climate system. But none of these questions may find an answer without acknowledging the moral connotations involved in these questions. I choose not to go in that direction and rather confine what we may learn from the present and past pandemics/epidemics.

In the case of MERS, yearly recurrent episodes of epidemics were registered since the first outbreak in 2012. Also here the CFR was very high, namely 36\% (690 fatalities of a total of 1936 WHO-confirmed cases) (Toosy and O'Sullivan 2017). An outspoken difference between MERS and 2019-nCoV is the limited distribution of the MERS epidemic, predominantly occurring in the Arabic peninsula, with one significant exception: this was the MERS outbreak in the Republic of (South) Korea in May-July 2015 (Toosy and O'Sullivan 2017). Nevertheless, MERS also caused victims in a large number of countries outside the region (Toosy and O'Sullivan 
2017). These data suggested that the MERS-related coronavirus was less infective, which may or not be attributable to a different entry of the virus (see Sect. 2).

Although the following results are still premature, recent studies at Erasmus MC in Rotterdam suggest that the increased infectivity of the $2019 \mathrm{nCoV}$, for instance compared with the SARS pandemic, may result from a much higher rate of virus shedding from the upper respiratory organs, especially from the nasal epithelium. Nearly 3 months after the outbreak of this new pandemic, it is way too early to draw firm conclusions from these observations. Also it is rather early to draw conclusions about a possible seasonal influence of the new virus. A possible seasonality dependence however has been suggested for other coronaviruses, e.g. causing the seasonal cold (like the Human coronavirus OC43), and the viruses causing influenza and other viral diseases (Martinez 2018). In a commentary paper, Cohen (2020) suggests that cold and dry air conditions, like in the ski-resorts in Northern Italy and other alpine regions, may have constituted ideal conditions for human-to-human infectivity (Cohen 2020). However, suggestions of the ideal humidity conditions in the air and airways, are very speculative and may constitute a dangerously biased surmise of protection. If physical parameters would offer such a degree of protection, by lowering the infectivity rate, this could only become noted after several seasons of the pandemic have passed.

Also the idea of acquiring immunity after recovering from the disease is a hopeful prospect, but a dangerously flawed idea for the risk groups with high CFR's (see Sect. 1). Without detailed knowledge of the etiology of the late immune responses causing vital organ failure and high numbers of fatalities, classical models do not answer these unfamiliar diseases (Allaerts 2019). Technically, models based on incorporating acquired-immunity during the spreading of an epidemic, may become flawed in the case of SARS and COVID-19, because of a misinterpretation of the totality of immune reactions involved (see Sect. 3). Whether or not alternative models based on different mathematical distributions (Allaerts 2019) are better suited, is too early to decide at this stage of the pandemic. Also the WHO-report (World Health Organization 2020) cautions, because, so far it is unknown how immunity against 2019-nCoV will become built up by the recovered patients. Besides, the potentially high risk of a frequently mutating virus-pool—changing its antigenic properties in the course of the seasons ahead-may dampen our optimism, despite the worldwide efforts to raise a vaccine against this new COVID-19. For comparison, the MERS epidemic resulted from a mutation in the pool of bat coronaviruses around 2012 (Toosy and O'Sullivan 2017), whereas also the new bat related coronaviruses SARS-CoV and SARS-CoV-2 appeared in a time span of only two decades (2002-2019).

\section{Summary of Questions for Further Research}

To summarize the present paper, a few highlights will be listed. However, this paper is not about how to organize a state of medical emergency, and neither about how to organize the production of masks and other protective clothing, nor about providing sufficient beds and electric fans for breathing machines. It is an attempt to 
summarize the present state-of-knowledge and in particular to understand the missing gaps in our knowledge of this pandemic. It appears that understanding this pandemic requires not only the knowledge of virologists, epidemiologists, intensive care physicians, immunologists and specialists of the interactions between the viral genomes and our human biochemistry, but the knowledge of all of them, together!

The following shortlist represents key areas where our limited knowledge should merit further scientific research: (1) correlation of previous medication use (especially ACE inhibitors) and disease progression in the groups of patients associated with high-comorbidity risks; (2) characterizing the immunological profile (multiple immunophenotyping, but also assessment of cytokine production profiles especially during early phases of the disease, prior to acute lung injury) of patients at risk for developing acute respiratory distress syndrome (ARDS); (3) influence of genomic differences between coronaviruses related to both structural (glyco)proteins and non-structural proteins, on the epidemiology and pathogenicity of these viruses, in particular on the infectivity, the speed of virus production and shedding and the biochemical entry routes with the host cells. Also the immunological reactions to these viral-host interactions by the host immune system are of primary importance. The latter route may also become very instructive for directing vaccine development to this 2019-nCoV.

Up to date, a lot of knowledge has been acquired about the etiology and severity of this pandemic, reflected by the CFR ratios, the age-distribution and comorbidities associated with its victims. Hopefully, science will come up with answers to questions regarding the underlying mechanisms too, in order to better protect public health.

\section{References}

Allaerts W (2019) Evaluating the connectivity, continuity and distance norm in mathematical models for community ecology, epidemiology and multicellular pathway prediction. In: 8th International conference on mathematical modeling in physical sciences, IOP Publ. Journal of Physics: Conference Series 1391:012119. https://doi.org/10.1088/1742-6596/1391/1/012119

Allaerts W, Chang TW (2017) Skewed exposure to environmental antigens complements hygiene hypothesis in explaining the rise of allergy. Acta Biotheor 65(2):117-134

Anthony SJ, Gilardi K, Menachery VD, Goldstein T, Ssebide B, Mbabazi R, Navarrete-Macias I, Liang E, Wells H, Hicks A, Petrosov A, Byarugaba DK, Debbink K, Dinnon KH, Scobey T, Randell SH, Yount BL, Cranfield M, Johnson CK, Baric RS, Lipkin WI, Mazet JAK (2017) Further evidence for bats as the evolutionary source of Middle East respiratory syndrome coronavirus. MBio 8(e00373-17). https://doi.org/10.1128/mBio.00373-17

Baumann AMT, Bakkers MJG, Buettner FFR, Hartmann M, Grove M, Langereis MA, de Groot RJ, Mühlenhoff M (2015) 9-O-Acetylation of sialic acids is catalyzed by CASD1 via a covalent acetyl-enzyme intermediate. Nat Commun 6:7673. https://doi.org/10.1038/ncomms8673

Chan JFW, Yuan S, Kok KH, To KKW, Chu H, Yang J, Xing F, Liu J, Yip CC, Poon RWS, Tsoi HW, Lo SKF, Chan KH, Poon VKM, Chan WM, Ip JD, Cai JP, Cheng VCC, Chen H, Yuen KY (2020) A familial cluster of pneumonia associated with the 2019 novel coronavirus indicating person-to-person transmission: a study of a family cluster. Lancet 3959(10223):514-523. https:// doi.org/10.1016/S0140-6736(20)30154-9)

Cohen J (2020) Why do dozens of diseases wax and wane with the seasons - and will COVID-19? Sciencemag (AAAS Newsletter). https://Sciencemag.org. Accessed 13 Mar 2020 
Ferrario CM, Jessup J, Chappell MC, Averill DB, Brosnihan KB, Tallant EA, Diz DI, Gallagher PE (2005) Effect of angiotensin-converting enzyme inhibition and angiotensin II receptor blockers on cardiac angiotensin-converting enzyme 2. Circulation 111:2605-2610

Guan W, Liang W, Zhao Y, Liang H, Chen Z, Li Y, Liu X, Chen R, Tang C, Wang T, Ou C, Li L, Chen P, Sang L, Wang W, Li J, Li C, Ou L, Cheng B, Xiong S, Ni Z, Hu Y, Xiang J, Liu L, Shan H, Lei C, Peng Y, Wei L, Liu Y, Hu Y, Peng P, Wang J, Liu J, Chen Z, Li G, Zheng Z, Qiu S, Luo J, Ye C, Zhu S, Cheng L, Ye F, Li S, Zheng J, Zhang N, Zhong N, He J (2020) Comorbidity and its impact on 1590 patients with COVID-19 in China: a nationwide analysis. medRxiv. https ://doi.org/10.1101/2020.02.25.20027664

Hamming I (2008) Novel insights in the pathogenesis of renal interstitial damage during ACE inhibition: a role for ACE2 and the (pro)renin-receptor? Groningen: University of Groningen (thesis)

Hoffmann M, Kleine-Weber H, Krüger N, Müller M, Drosten C, Pöhlmann S (2020) The novel coronavirus 2019 (2019-nCoV) uses the SARS-coronavirus receptor ACE2 and the cellular protease TMPRSS2 for entry into target cells. bioRxiv 2019:69

Holt PG (1994) The role of dendritic cells in immune defense of the airways. In: Bruijnzeel-Koomen CAFM, Hoefsmit ECM (eds) Immunopharmacology of macrophages and other antigen-presenting cells. Academic Press, London, pp 99-115

Imai Y, Kuba K, Penninger JM (2008) The discovery of angiotensin-converting enzyme 2 and its role in acute lung injury in mice. Exp Physiol 93(5):543-548

International Committee on Taxonomy of Viruses (2012) Virus taxonomy: ninth report of the international committee on taxonomy of viruses part II. The positive sense single stranded RNA viruses (Family Coronaviridae). Elsevier, New York

Lau SKP, Feng Y, Chen H, Luk HKH, Yang WH, Li KSM, Zhang YZ, Huang Y, Song ZZ, Chow WN, Fan RYY, Ahmed SS, Yeung HC, Lam CSF, Cai JP, Wong SSY, Chan JFW, Yuen KY, Zhang HL, Woo PCY (2015) Severe acute respiratory syndrome (SARS) coronavirus ORF8 protein is acquired from SARS-related coronavirus from greater horseshoe bats through recombination. J Virol 89(20):10532-10547

Martinez ME (2018) The calendar of epidemics: seasonal cycles of infectious diseases. PLoS Pathog 14(11):e1007327. https://doi.org/10.1371/journal.ppat.1007327

Oudit GY, Kassiri Z, Jiang C, Liu PP, Poutanen SM, Penninger JM, Butany J (2009) SARS-coronavirus modulation of myocardial ACE2 expression and inflammation in patients with SARS. Eur J Clin Invest 39:618-625

Raj VS, Mou H, Smits SL, Dekkers DHW, Müller MA, Dijkman R, Muth D, Demmers JAA, Zaki A, Fouchier RAM, Thiel V, Drosten C, Rottier PJM, Osterhaus ADME, Bosch BJ, Haagmans BL (2013) Dipeptidyl peptidase 4 is a functional receptor for the emerging human coronavirusEMC. Nature 495(7440):251-254

Rubenfeld GD, Caldwell E, Peabody E, Weaver J, Martin DP, Neff M, Stern EJ, Hudson LD (2005) Incidence and outcomes of acute lung injury. N Engl J Med 353(16):1685-1693

Salamatbakhsh M, Mobaraki K, Sadeghimohammadi S, Ahmadzaeh J (2019) The global burden of premature mortality due to the Middle East respiratory syndrome (MERS) using standard expected years of life lost, 2012 to 2019. BMC Public Health 19:1523. https://doi.org/10.1186/ s12889-019-7899-2

Sommerstein R (2020) Re: Preventing a covid-19 pandemic: ACE inhibitors as a potential risk factor for fatal Covid-19 (Rapid Response to Editorial: Preventing a covid-19 pandemic). BMJ 368:810. https://doi.org/10.1136/bmj.m810

The Novel Coronavirus Pneumonia Emergency Response Epidemiology Team (2020) The epidemiological characteristics of an outbreak of 2019 novel coronavirus diseases (COVID-19)—China, 2020. China CDC Weekly 2(8):113-122

Toosy AH, O'Sullivan S (2017) An overview of middle east respiratory syndrome in the middle east. Emerg Chang Infect Dis. Elsevier, New York

Turner AJ (2015) ACE2 cell biology, regulation, and physiological functions. The protective arm of the renin-angiotensin system (RAS). Elsevier, New York. https://doi.org/10.1016/B978-0-12801364-9.00025-0

Weiss SR, Leibowitz JL (2011) Coronavirus pathogenesis. Adv Virus Res 81:85-164

World Health Organization (2020) Report of the WHO-China Joint Mission on Coronavirus Disease 2019 (COVID-19). https://www.who.int. 16-24 Feb 2020

Wu A, Peng Y, Huang B, Ding X, Wang X, Niu P, Meng J, Zhu Z, Zhang Z, Wang J, Sheng J, Quan L, Xia Z, Tan W, Cheng G, Jiang T (2020) Genome composition and divergence of the novel 
coronavirus (2019-nCoV) Originating in China. Cell Host Microbe 27:325-328. https://doi. org/10.1016/j.chom.2020.02.001

Publisher's Note Springer Nature remains neutral with regard to jurisdictional claims in published maps and institutional affiliations. 